\title{
Reduced hepatic microsomal triglyceride transfer protein (MTP) expression in a mouse model of non-alcoholic steatohepatitis (NASH) induced by choline-deficient high fat diet (CDAHFD)
}

Noriaki Kume, Daisuke Yasuda, Haruki Torii, Yoshinori Hiraoka

Division of Clinical Pharmacy, Faculty of Pharmaceutical Sciences, Kobe Gakuin University, Kobe 650-8586, Japan

Background: Non-alcoholic steatohepatitis (NASH) is a major health problem based upon abdominal obesity and metabolic syndrome. We, previously, have shown that serum total cholesterol (T-cho) and triglyceride (TG) levels, as well as body weight (BW), were significantly decreased in a choline-deficient high fat diet (CDAHFD)-induced NASH mice. We, therefore, have explored the molecular mechanisms involved in the reduced serum lipid levels.

Methods: C57BL/6J male mice (SPF, 8 weeks old) were fed CDAHFD or standard diet (SD, 10 mice each) for 14 weeks. Serum lipid profiles, liver histology, hepatic TG and expression of genes involved in lipid metabolism and $\mathrm{NASH}$ in the liver were measured, and their correlations were explored. Results:

Figure 1.

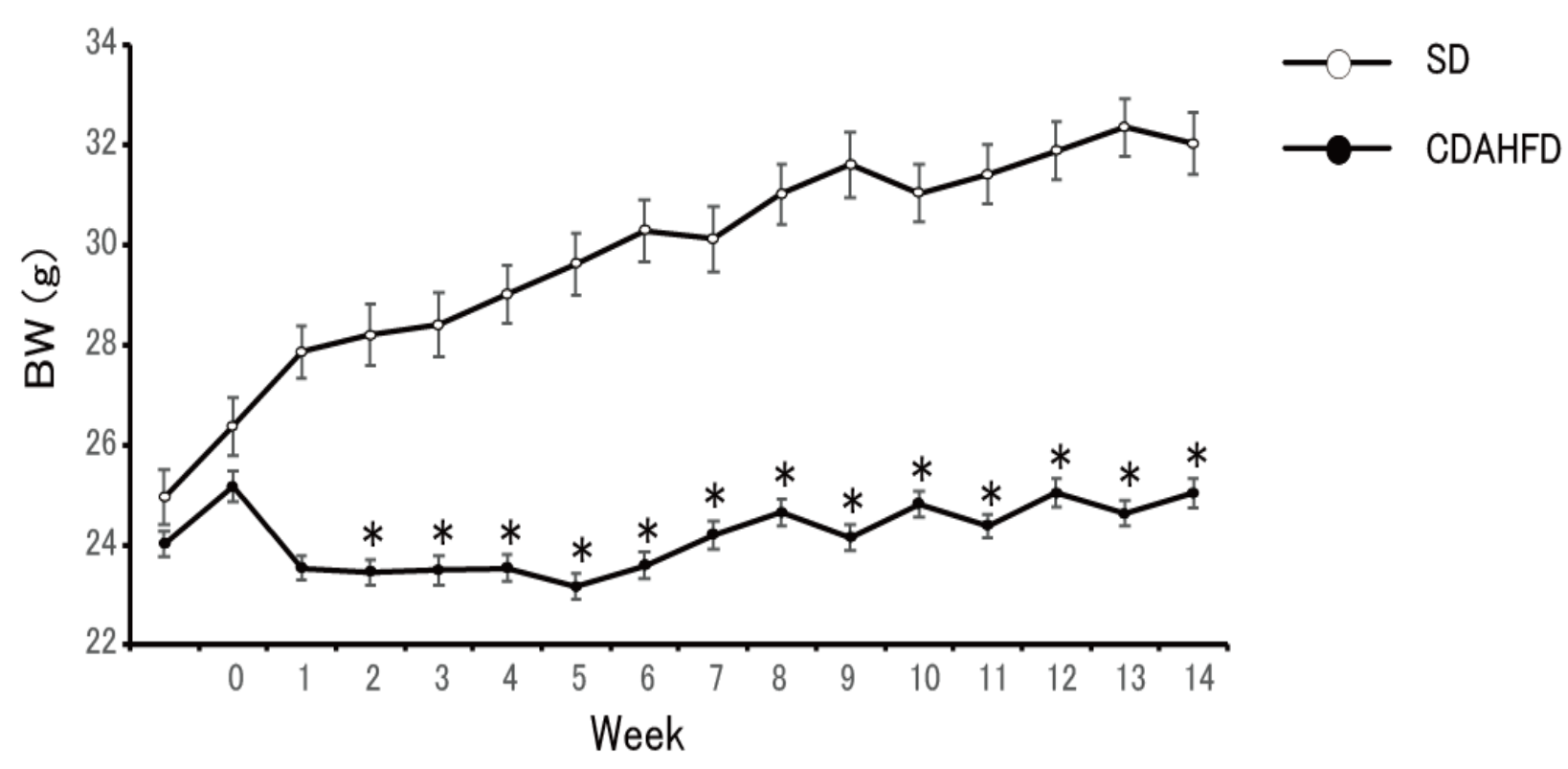

Body weigh was significantly lower in mice fed CDAHFD than in those fed SD.

Figure 2.
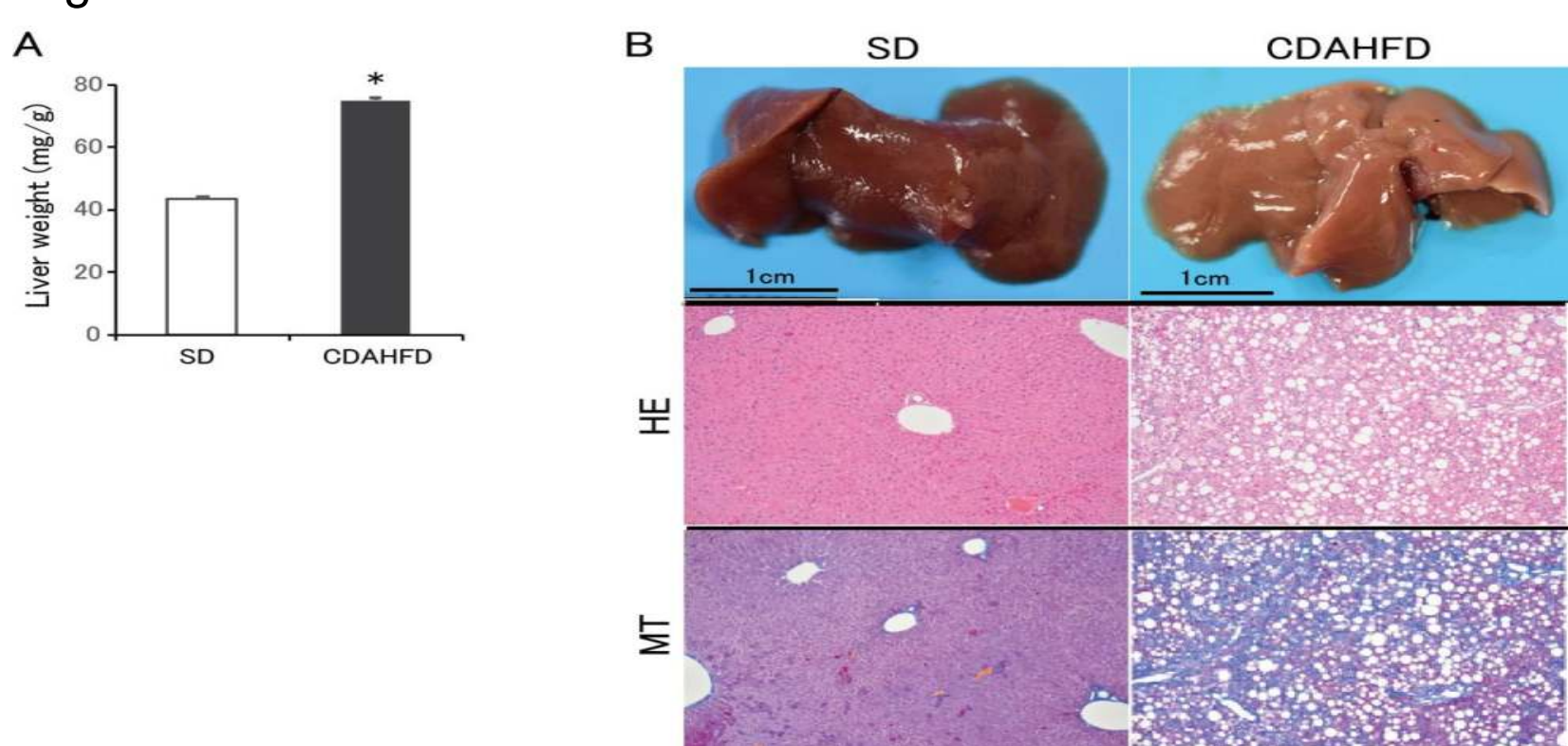

Livers in mice fed CDAHFD, but not SD, showed remarkable lipid accumulation and fibrosis which are characteristics of $\mathrm{NASH}$.

\section{Summary:}

Hepatic MTP expression was significantly suppressed in mice fed CDAHFD, and tended to be inversely correlated to NASH.

\section{Conclusion:}

A mouse CDAHFD model has several aspects distinct from human $\mathrm{NASH}$ based upon abdominal obesity, and may represent, in part, a mouse model of NASH induced by MTP inhibitors,
Figure 3.
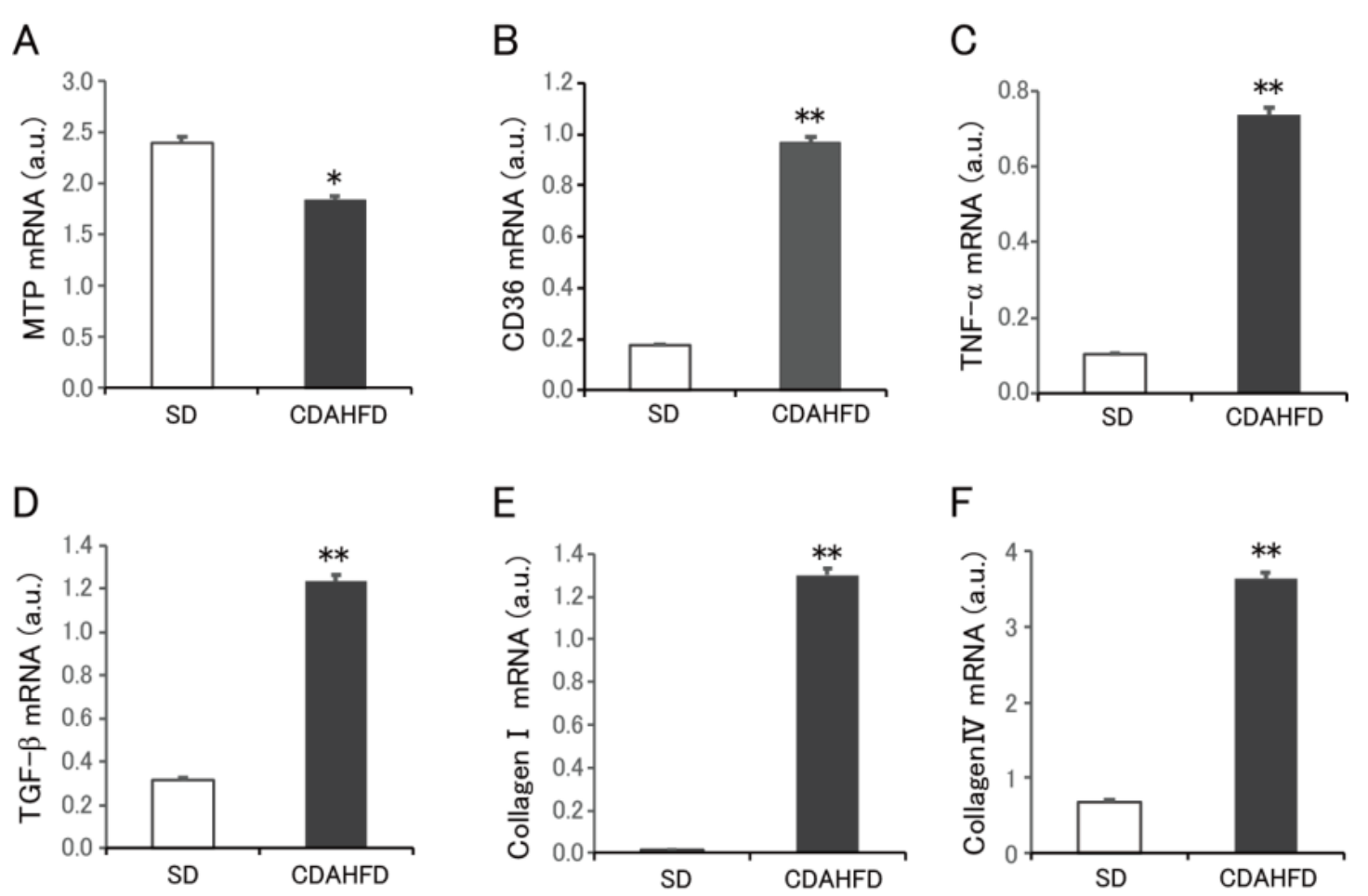

Hepatic MTP-1 expression was significantly suppressed, although hepatic CD36, TNF- $\alpha$, TGF- $\beta$ and collagen I expression was significantly increased, in mice fed CDAHFD than those fed SD.

Figure 4.
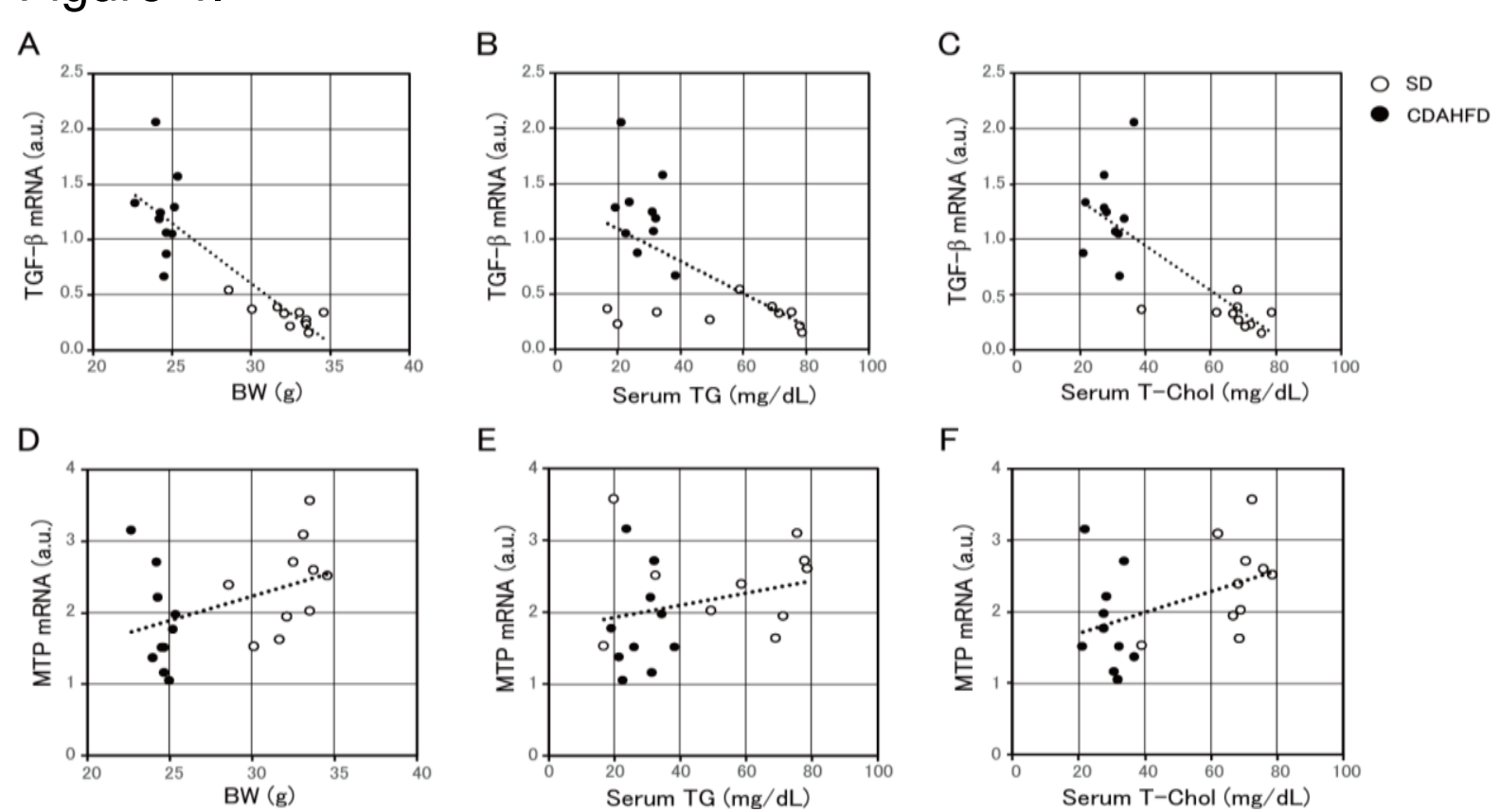

MTP expression tended to be positively correlated with BW and serum TG and T-cho levels, although hepatic TGF- $\beta$ expression showed statistically significant inverse correlation with them.

Figure 5.
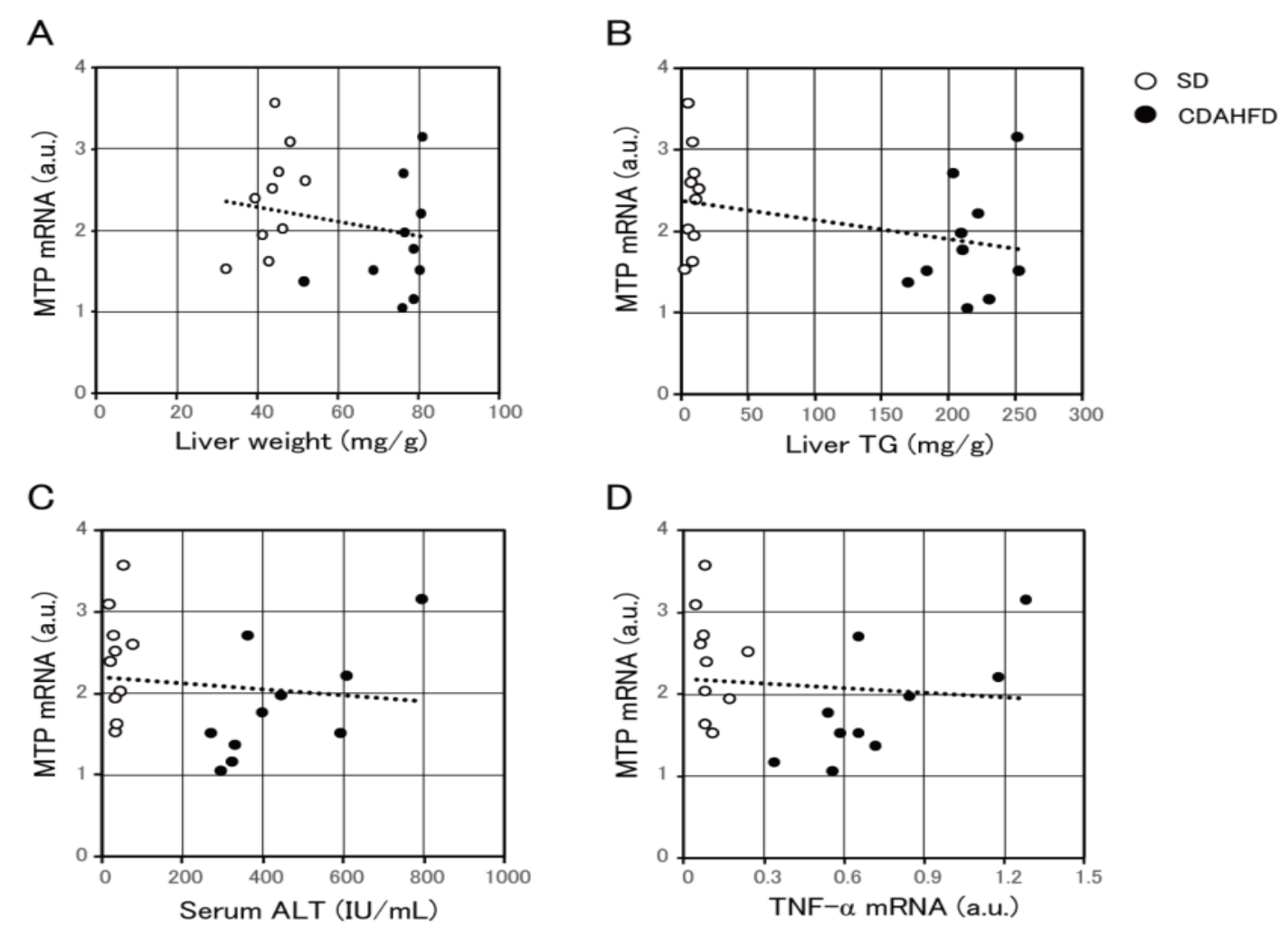

MTP expression tended to be inversely correlated with NASH phenotypes, such as liver weight, liver TG levels and TNF- $\alpha$ mRNA expression and serum ALT levels. 